\title{
Advancing biotechnology with CRISPR/Cas9: recent applications and patent landscape
}

\author{
Raphael Ferreira $^{1,2} \cdot$ Florian David $^{1,2} \cdot$ Jens Nielsen $^{1,2,3}$ (D)
}

Received: 7 November 2017 / Accepted: 19 December 2017 / Published online: 24 January 2018

(c) The Author(s) 2018. This article is an open access publication

\begin{abstract}
Clustered regularly interspaced short palindromic repeats (CRISPR) is poised to become one of the key scientific discoveries of the twenty-first century. Originating from prokaryotic and archaeal immune systems to counter phage invasions, CRISPRbased applications have been tailored for manipulating a broad range of living organisms. From the different elucidated types of CRISPR mechanisms, the type II system adapted from Streptococcus pyogenes has been the most exploited as a tool for genome engineering and gene regulation. In this review, we describe the different applications of CRISPR/Cas9 technology in the industrial biotechnology field. Next, we detail the current status of the patent landscape, highlighting its exploitation through different companies, and conclude with future perspectives of this technology.
\end{abstract}

\section{Introduction}

Throughout the years following the discovery of the structure of DNA, scientists have endeavored to genetically manipulate organisms. Until recently, most of the genetic engineering tools developed were based on DNA:protein recognition principles, such as restriction enzymes, sitedirected zinc finger nucleases (ZFs), and TAL effector nucleases (TALENs) $[6,47]$. However, these tools are commonly experienced with difficulties in design, synthesis, and efficiency which altogether prevented a global widespread adoption, e.g. TALENs require 30-35 amino acids repeats, each only recognizing a single nucleotide (nt) [29]. On the other hand, the RNA-programmable CRISPR/Cas9 technology has led to a scientific revolution by solving all of the above-mentioned issues [20]. The technology relies on two elements: a protein, the CRISPR associated protein

Jens Nielsen

nielsenj@chalmers.se

1 Department of Biology and Biological Engineering, Chalmers University of Technology, 41296 Göteborg, Sweden

2 Novo Nordisk Foundation Center for Biosustainability, Chalmers University of Technology, 41296 Göteborg, Sweden

3 Novo Nordisk Foundation Center for Biosustainability, Technical University of Denmark, 2800 Kgs. Lyngby, Denmark
(Cas9), and a RNA molecule, the guide RNA (gRNA) [5, 46]. Cas9, the first Cas protein used in genome editing, is a large multi-domain enzyme interacting with the gRNA, the target DNA, and the Protospacer Adjacent Motif sequence (PAM) (Fig. 1a). The gRNA element is composed of two distinct elements: the spacer, a $20 \mathrm{nt}$ domain that binds to the DNA; and the scaffold, a $\sim 79$ nt domain that interacts with Cas9 (Fig. 1a). Once guided to the target, Cas9 catalytically cleaves the DNA sequence $3 \mathrm{nt}$ upstream the 5'-NGG PAM, resulting in the activation of endogenous repair mechanisms, such as homologous recombination (HR) or non-homologous end joining (NHEJ) [2, 71] (Fig. 1b).

\section{Expanding Cas9 features through enzyme engineering}

The structural characterization of Cas9 has led to the development of mutagenized variants with various catalytic properties, specificities through different PAM recognition preferences and reduction in off-targeting [37, 55, 56, 90]. For example, Hirano et al. first characterized FnCas 9 from Francisella novicida and, based on the characterized protein structure, created a variant recognizing a 5'-YG' PAM instead of the original 5'-NGG [37]. A 5'-YG PAM increases the target space availability for genome editing, i.e. any target followed by CG or TG is prone to be targeted by the gRNA:FnCas9 complex. Additionally, other CRISPR nucleases with different PAM preferences can also be used to increase the target space availability, e.g. FnCpf1 
A

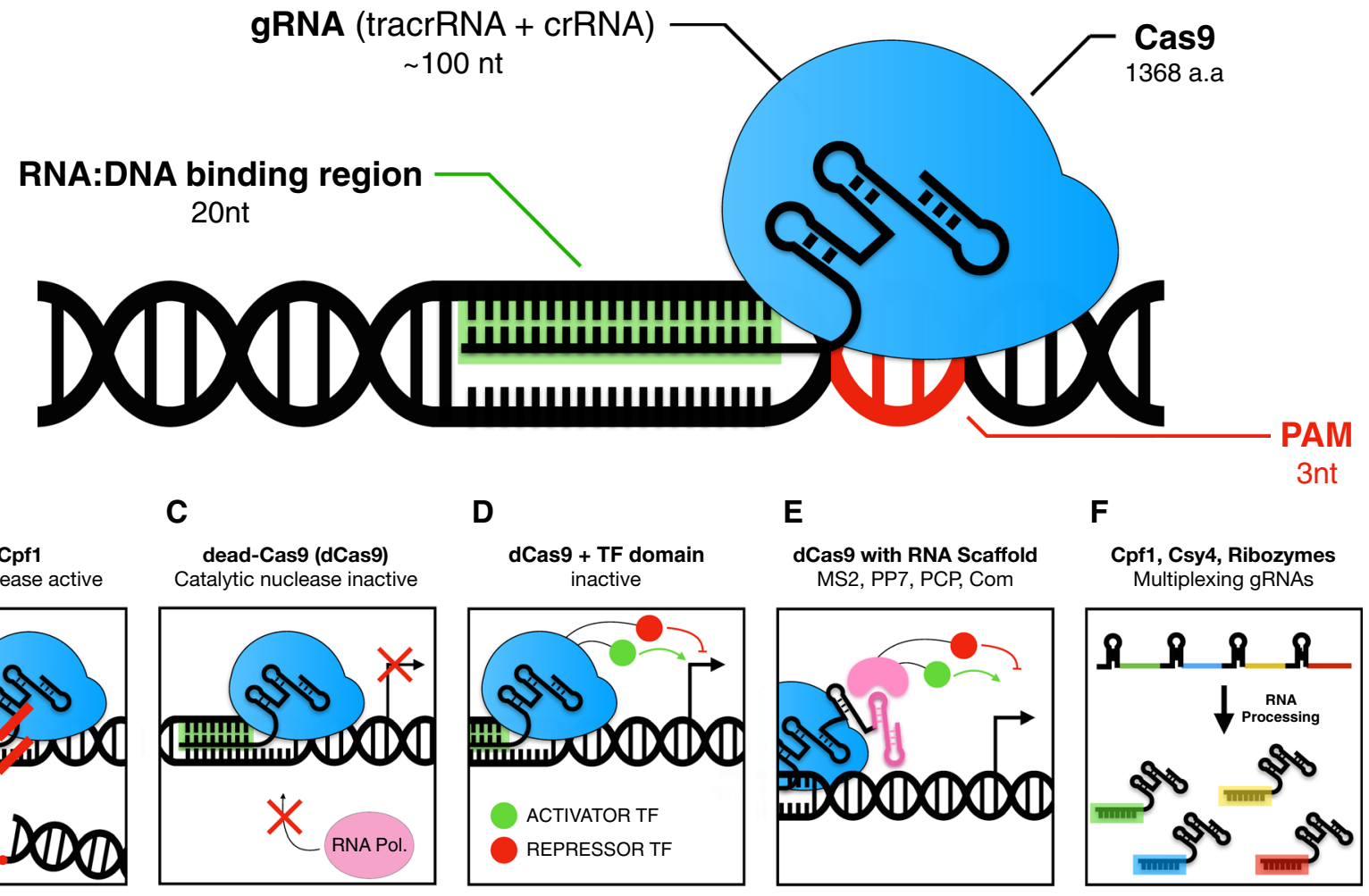

Fig. 1 CRISPR/Cas9 for genome editing and gene regulation. a The gRNA:Cas9 complex binding to the DNA target. In green, the spacer region which interacts with the DNA target. b DNA cut generated from the Cas9 nuclease activity. c Gene regulation with dCas9 physically blocking the RNA polymerase from binding to the promoter

a type V CRISPR system from $F$. novicida which recognizes a T-rich PAM 5'-TTTN [26, 101] (Fig. 1b). In another approach, mutation in one of the nuclease activity domains (RuvC ${ }^{\mathrm{D} 10 \mathrm{~A}}$ or $\mathrm{HNH}^{\mathrm{H} 840 \mathrm{~A}}$, Cas9n) was shown to result in a modified Cas9 only capable of performing single-strand DNA breaks (nick) instead of the original blunt DNA break [80]. This feature has been shown to reduce off-targeting and enhance HR in some organisms [12, 70]. By extension, 'paired nickases', i.e. using two adjacent gRNAs with Cas9n, can efficiently introduce both indel mutations and HR events with a single-stranded DNA oligo-nucleotide donor template in mammalian cells $[28,10,80]$. Complete disruption of the endonuclease activities (RuvC ${ }^{\mathrm{D} 10 \mathrm{~A}}$ along with $\mathrm{HNH}^{\mathrm{H} 840 \mathrm{~A}}$ ) results in a catalytically inactive Cas9, or dead-Cas9 (dCas9) $[78,79]$. This has been exploited to physically block the transcriptional machinery when targeted in the promoter region of a gene of interest, coined CRISPR interference (CRISPRi) [22, 34] (Fig. 1c). Additionally, repression can be further enhanced by fusing dCas9 with repressive domains, such as the mammalian transcriptional repressor domain Mxi1 [33] (Fig. 1d). Gander et al. have recently exploited dCas9-Mxi1 repressive mechanism to effectively built up to seven layers of synthetic NOR gate circuits, in S. cerevisiae region. d CRISPR interference further enhanced with dCas9 fused with transcriptional regulators. e gRNA scaffold extended with stemloops recruiting regulator elements. f. Multiplexing gRNAs from a single transcript through endoribonuclease or self-processing elements

[30] (Figs. 1d, 2b). Likewise, dCas9 can be coupled to activating transcription factor domains, such as the tripartite VP64-p65-Rta (VPR) or the RNAP $\omega$-subunit (rpoZ), which have been characterized as powerful tools for activating genes [4, 7, 44, 91] (Fig. 1d). Similarly, epigenetic regulators, such as methylation, demethylation, acetylation and deacetylation domains, can be fused to dCas9 to influence chromatin structure and, therefore, interfere with the transcriptional signature of a promoter [36, 50,54]. Hilton et al. reported the fusion of dCas9 with the histone acetyltransferase domain of the human E1A-associated protein $\mathrm{p} 300$ (dCas9-p300), which significantly modulated the chromatin structure, and resulted in a 4000-fold up regulation with a single gRNA [36].

\section{The gRNA characteristics and extensions}

Cas9 can be guided virtually anywhere in the genome where a PAM sequence is present. However, several parameters, such as nucleotide motifs, particularity of the PAM sequence, and mismatches in the guide, have to be taken into account for a correct cleavage in the target DNA [91]. Recently, efforts have been made to solve 

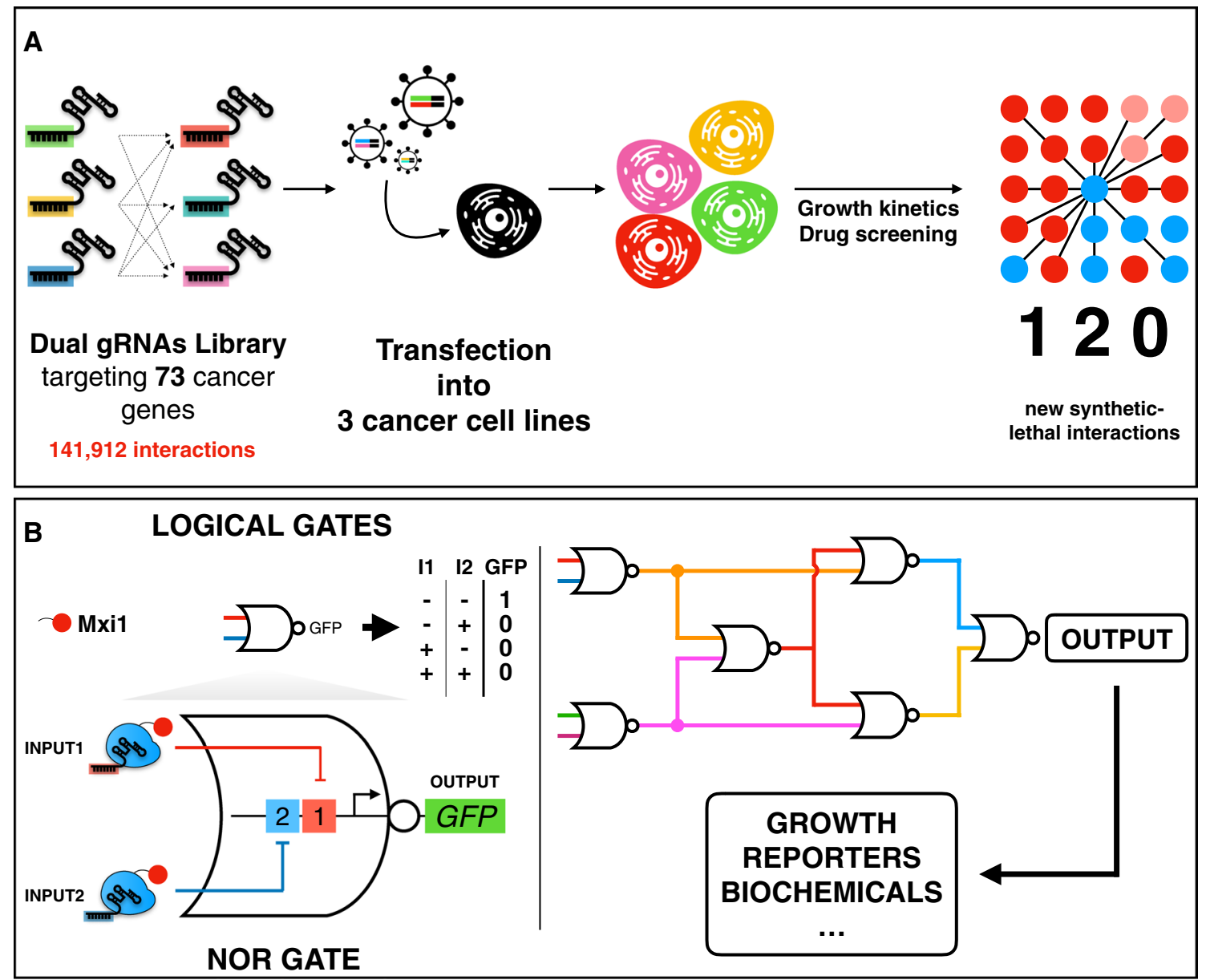

Fig. 2 Example of application in drug discovery and synthetic biology. a Genome-wide pooled gRNA libraries targeting all pairs of 73 cancer genes with dual guide RNAs in three mammalian cell lines.

target efficiency through algorithms predicting the ability of the gRNA to facilitate DNA cleavage by Cas9 at the intended target site by employing refined machine learning methods and incorporating large training datasets [18, 19]. The sequence accuracy of the gRNA is also essential to achieve a correct base-pairing between the gRNA and the target DNA. Most commonly, RNA pol. III promoters are used to transcribe gRNAs. These are scarce and more importantly, some of them contain idiosyncratic features, e.g. U6 mammalian promoter requires to have a $\mathrm{G}$ at the $5^{\prime}$ end of the transcript [28]. Transcriptional expression can be improved by inserting self-processing elements, such as HDV ribozyme and tRNAs, at the $5^{\prime}$ or $3^{\prime}$ end to prevent potential degradation of the transcript $[49,83]$. Processing elements can also be exploited to multiplex several gRNAs in a row by collocating those element between each gRNAs [17] (Fig. 1e). Several examples, such as the type III CRISPR-Csy4 [25, 76] or natural CRISPR array $[1,14]$ have been shown to efficiently generate multiple b Example of logic circuits made with dCas9-Mxi NOR gates with GFP signal used as output similar to Gander et al. study

gRNAs from a single transcript (Fig. 1e). Notably, while Cpf1 belongs to the same CRISPR class II as Cas9, i.e. only a single crRNA-effector enzyme and no tracrRNA part is required for cutting DNA. It differs from it by possessing a specific RNA processing domain that allows to process the crRNA into multiple gRNAs [55, 69, 92, 101].

Finally, the gRNA scaffold can be extended to include effector protein recruitment stem-loops, which has been shown to enhance transcriptional regulation $[8,44,100]$ (Fig. 1d). With that strategy, Zalatan et al. were able to design gRNAs to either recruit activator or repressor elements, which ultimately established both, repression and activation of specific gene targets at the same time [100] (Fig. 1d). This platform offers a considerable advantage in comparison to dCas9 fused to a regulator, as it is not limited to which transcriptional regulator is fused to dCas9, but to which stem-loop is connected to the gRNA scaffold.

Another high potential application area for the CRISPR technology is systematic genetic screening 










employing gRNA libraries. Due to the short length of gRNAs ( 100nt), accurate predictability, and easy cloning approaches, genome-wide gRNA libraries have been successfully designed to knockout and regulate genes throughout the entire genome [31]. For example, Shen et al. developed a systematic approach to map synthetic lethality genes by targeting all pairs of 73 cancer genes with dual guide RNAs in three different cancer cell lines (Fig. 2a). Their strategy involved nine gRNA pairs per combination, the library comprised 23,652 double-geneknockout constructs with two replicates in three cell lines which ultimately led to a total of 141,912 interactions and to the discovery of 120 potential drugs candidates [86] (Fig. 2a).

\section{Industrial applications through metabolic engineering}

\section{Genome engineering}

There has been an increasing interest in improving microbial cell factories through metabolic engineering approaches using CRISPR/Cas9 technology [42]. The efficiency and versatility offered by CRISPR tools have shown great potential in rewiring the metabolic network of host cells to enhance their production of metabolites used in various areas of industrial biotech ranging from applications as biofuels to chemical building blocks and pharmaceuticals (Table 1). Metabolic pathway optimization towards the product of interest commonly requires deletions of multiple genes, e.g. competitive metabolic pathways, which is traditionally performed through iterative cycles of genetic marker integration and removal [15]. Conversely, the CRISPR technology does not necessitate integrative markers, and several efficient markerfree approaches were developed to perform multiplexed genome editing, e.g. knockouts, point-mutations [41, 93] and gene integration [45], which extensively reduced the time and effort required to perform targeted strain engineering. The CRISPR technology has also improved genetic engineering in difficult-to-engineer industrial organisms, such as food crops. Among several examples (Table 1), Li et al. reported a significant site-specific gene replacement of the 5-enolpyruvylshikimate-3-phosphate synthase $(E P S P S)$ in rice plants using a pair of gRNAs targeting introns, ultimately converting the crop into a glyphosate-resistant one (Fig. 3c) [62]. More recently, several studies have highlighted significant improvements in genome editing in plants using DNA-free CRISPR/Cas9 ribonucleoproteins [65, 98].

Besides its multiplexing qualities, CRISPR has also shown great efficiency to integrate large pathways and 
libraries $[38,88]$. For example, Shi et al. specifically designed gRNAs to target multiple delta sites in the yeast genome, ultimately achieving 18-copy genomic integrations of a $24 \mathrm{~kb}$ combined xylose utilization and (R,R)2,3-butanediol (BDO) production pathway in a single step, in $S$. cerevisiae [88]. DNA libraries, such as error-prone PCRs derived or double-stranded fragments obtained from DNA synthesizing companies, can be genomically integrated to find variants of a studied enzyme with enhanced catalytic activities or optimal level of expression [64, 83]. Genomically integrated DNA libraries offer several advantages compared to plasmid based strategies, especially in terms of expression stability [83]. Liang et al. used that strategy to integrate 640 ribosome binding sites (RBS) for five different enzymes involved in the production of isopropanol in E. coli [64] (Fig. 3a). After multiple rounds of screening for strains carrying the best RBS variants, i.e. optimal expression of the gene leading to a higher isopropanol titer, a final strain producing $7.1 \mathrm{~g} \mathrm{~L}^{-1}$ of isopropanol was obtained [64].

\section{dCas9-transcriptional regulation}

Fine-tuning of biosynthetic pathways is a key step in the correct and efficient synthesis of a particular target compound (Nielsen and Keasling 2016). Traditional strategies have been relying on a limited number of characterized promoters to control gene expression, i.e. strong, weak, and inducible promoters [51]. As mentioned above, besides its efficient endonuclease activity, CRISPR can enable gene expression modulation through the deactivated form of the Cas9 protein, dCas9 $[59,79]$. Once bound to, or in the vicinity of the transcriptional start site (TSS), the gRNA:dCas9 complex can significantly alter the transcriptional expression by physically interfering with RNA polymerase binding $[14,43,79]$. Wu et al. recently exploited this strategy in $E$. coli where they did a selective knockdown of gene expression of enzymes that could divert the carbon flux away from the production of 1,4-Butanediol (BDO) [99]. They divided their study into two phases, (1) a heavy strain engineering approach through multiple genome edits such as gene knockouts, knockins, and point-mutations, and (2) optimization through fine tuning of gene expression of three genes competing with the production of BDO. This strategy increased the 1,4-BDO titer by $100 \%$ from phase (1) to phase (2), resulting in a final titer of $1.8 \mathrm{~g} \mathrm{~L}^{-1} 1,4-\mathrm{BDO}$ (Table 1).

In addition, graded transcriptional patterns can be achieved depending on where the $\mathrm{dCas} 9$ complex binds in the promoter region, e.g. on the TSS for strong downregulation or more distanced from it for a medium repression. Thus, optimal gene expression can be elucidated by targeting dCas9 at different positions on the studied promoter $[16,17,44]$. This feature is subject to several parameters, such as the distance to TSS, condition dependent presence of transcription factors, chromatin accessibility, but the complete understanding of how to obtain precise regulation has yet to be characterized and is most likely dependent on specific promoters [57, 91]. For example, Deaner et al. recently developed a graded expression platform that can be employed to systematically test enzyme perturbation sensitivities (STEPS), and assists to identify potential flux limiting enzymes arising from production pathways [16] (Fig. 3d; Table 1). Their strategy relied on targeting dCas9, with either a repressor and activator domain, at different positions of several promoters of genes, and analyze their effect on the final titer. For example, while optimizing glycerol production, from the seven tested genes, one gRNA targeting GPD1 with dCas9-VPR led to a significant titer increase, highlighting its importance in the overall production pathway. Then they iteratively used STEPS to find a second bottleneck in $G P P 1$, which ultimately led to a final titer $\sim 28 \mathrm{~g} \mathrm{~L}^{-1}$, a sevenfold increase compared with their original strain.

\section{Patenting landscape}

The patent landscape related to CRISPR/Cas9 technology is complex, constantly changing, with several main actors dominating the field [21]. Those include one hospital, five universities, and one researcher, namely: Massachusetts General Hospital, Duke University, the Broad Institute (joint Harvard and MIT entity), the University of California Berkeley, the University of Vienna and Emmanuelle Charpentier. These entities have granted broad exclusive licenses to "surrogate" companies such as Caribou Bioscience (Berkeley, Vienna University, Jennifer Doudna), CRISPR Therapeutics (E. Charpentier; therapeutic field) and ERS genomics (E. Charpentier; all applications, except human therapeutics). Additionally, several spin-out companies have been formed, e.g. Editas Medicine (Broad institute, Duke University, Massachusetts General Hospital; area of human therapeutics) and Intellia Therapeutics (Caribou Biosciences; human therapeutics) with focus on their own R\&D activities in human therapy, and specific out-licensing in certain areas. Notably, Editas Medicine, CRISPR Therapeutics and Intellia Therapeutics are publicly registered in the NASDAQ Stock Market.

Regarding the different commercialization areas of these patents, three main application fields have formed: (1) CRISPR/Cas9 used in medical applications with focus on human therapeutics and drug discovery, (2) research tool applications, cell line and animal models, and (3) agriculture and food applications (Fig. 4). 


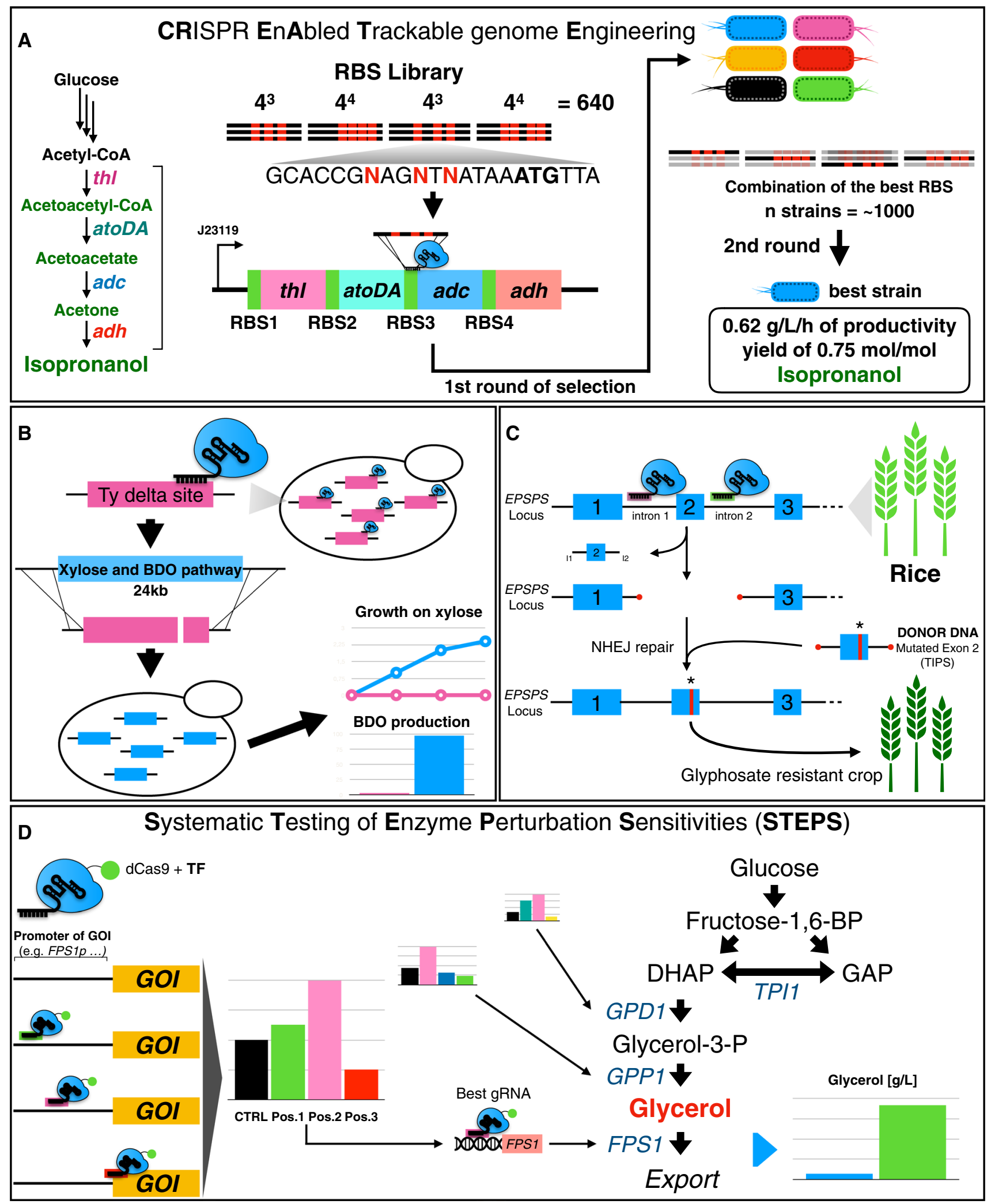


4Fig. 3 CRISPR applications in metabolic engineering. a CRISPR enabled trackable genome engineering (CREATE) strategy for optimal expression of 4 genes involved in isopropanol biosynthetic pathway. Integration of DNA libraries composed of variant RBS sequences. b Multiple integration of the xylose and BDO pathway into retroposons sites in S. cerevisiae. Retrotransposons are composed of similar DNA sequences, which ultimately allows to generate a promiscuous gRNA able to target several of these targets at once. c Genetic engineering in rice using dual gRNAs targeting ESPS introns for double amino acid substitution [T102I + P106S (TIPS)]. d Systematic testing of enzyme perturbation sensitivities (STEPS) approach to iteratively find bottleneck

In the area of human therapeutics, spin-outs originated from academic institutions and initial inventors are dominating the field with focus on R\&D, licensing, and commercial partnering. We see specific exclusive licenses to newly formed companies in the field, e.g. for Chimeric Antigen Receptor T-cell therapy (CAR-T) (Juno, Novartis and Cellectis) or treatment of blood, eye and heart diseases (Casebia, and Editas Medicine), as well as broader licenses in using CRISPR as a drug for human therapeutics (AstraZenenca, Amri, Oxford Genetics and Evotec) (Figs. 4, 5).

In the area of research tools, non-exclusive licenses, mostly coming from the Broad Institute and Caribou Biosciences, are most prominent in the field. The applications range from licenses for general research tools, e.g. Clontech, Horizon, ATCC, GE-Healthcare, to specific licenses in the field of drug discovery, e.g. Evotec, Novartis, Regeron, and applications in animal models, e.g. Taconic, Sage Labs, The Jackson Laboratory, and Knudra (Fig. 4).

In the area of agricultural and food applications, larger industry players, such as DowDupont, control the field with regards to patent holding and licensing. Their strategy included (1) the acquisition of Danisco in 2011, an agricultural/food ingredient company that made crucial progress in understanding CRISPR mechanism and the role of Cas9 [2], (2) agreements with Virginijus Siksnys from University of Vilnius, one of the founders of CRISPR technology $[32,84]$, and (3) exclusive cross-licenses from Caribou Bioscience and ERS Genomics specific for the agricultural field. In addition, Monsanto/Bayer Crop Science recently acquired a non-exclusive license from the Broad Institute for sole use in the agricultural sector. Another key player in the field of crop engineering is Calyxt, which acquired exclusive worldwide rights for CRISPR/Cas9 utilization in plants from the University of Minnesota, highlighting the complexity emerging from these patents and the different licensing structures in the field.

In the area of industrial biotechnology, CRISPR licenses are so far only obtained in a small number of cases, such as Evolva which acquired a license from ERS genomics for yeast and fungal engineering for biotechnological production of chemicals.
Because of the ongoing patent dispute between the Broad Institute and UC Berkeley/Charpentier, the licensing situation remains opaque. Currently, some of the Broad patents were granted in the beginning of 2017 while the UC Berkeley/E. Charpentier patents are still pending. A request of interference filed by UC Berkeley was turned down in the first round but has now gone to a second round with an appeal to the original decision. The hubbub created by the "battle" has incentivized several companies, e.g. Horizon, DowDupont, Sage labs, to acquire licenses from different main patent owners, to secure even exclusive access to the technology in a certain field.

A main area in CRISPR-based drug development is its use in cancer immunotherapy to reprogram enhanced CAR-T receptors for selectively targeting cancer cells [81]. The genetic modifications are done in vitro, making this approach a potential low hanging fruit for successful approval of CRISPR based medical therapies. A major milestone was recently achieved with two CAR-T based treatments approved by the FDA [73, 74]. Large companies and several startups acquired exclusive licensing from different CRISPR IP holders in the field, e.g. Novartis with Intellia Therapeutics, and Juno with Editas Medicine (Fig. 4).

Looking at all the different patents and patent applications in the field, the total number of patents encompasses over 90 granted patents and 1300 filed patents ranging from CRISPR/Cas9 components to delivery systems and applications [21]. Some of the main actors started to create patent pools to simplify the licensing process for commercial users. As such, agreements were made between CRISPR Therapeutics, Intellia Therapeutics, Caribou Biosciences and ERS Genomics to maintain and coordinate prosecution of particular patent families. As direct competitors, a similar alliance has been formed between the Broad Institute, Rockefeller University, Harvard University, and MIT, through the intermediary of MPEG LA, LLC firm Sheridan [87].

Another strategy followed by certain entities in the field is to diversify their IP portfolio with the result of having priority for follow-on refinements of the previous patent applications. For example, Zhang and colleagues from the Broad Institute have discovered and filed patent protection for $\mathrm{Cpf1}$, a robust alternative to Cas9 [21, 101].

\section{Conclusion and discussion}

Only recently discovered, CRISPR/Cas9 technology has already been enhanced to the point of fulfilling most of the genome editing and gene regulation currently demanded, ranging from the ability to perform multiple gene insertions, gene knockouts, combinatorial libraries, to advanced fine-tuning of biosynthetic pathways [23, 39, 47, 95]. However, off-targeting remains an important limitation to the 


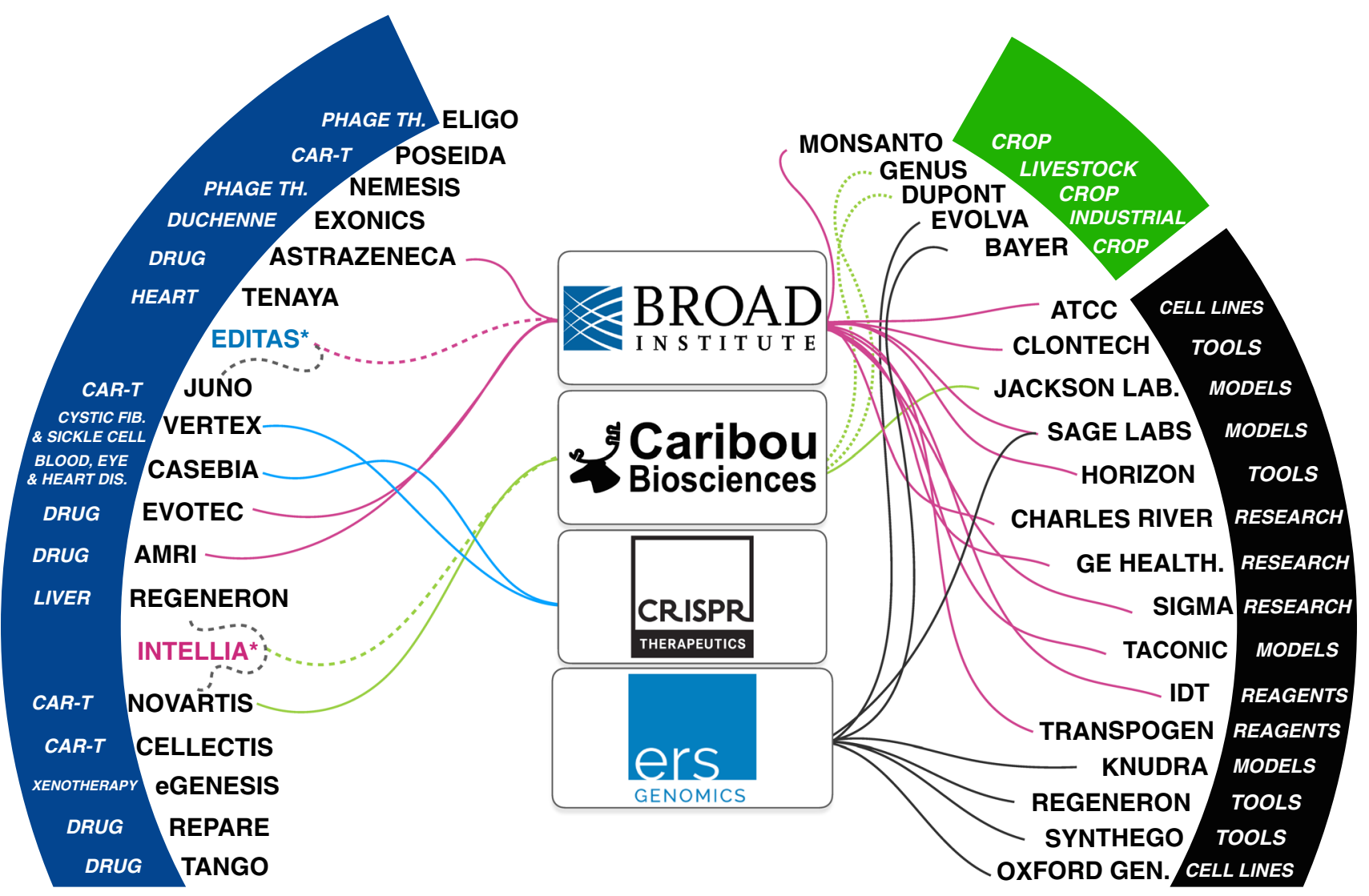

Fig. 4 CRISPR companies and licensing agreements. Bold lines represent non-exclusive licensing. Dashed lines represent exclusive licensing. In the middle, the four most important owners of CRISPR patents. In dark blue, companies applying CRISPR for health-related

technology, with several studies pointing out unwanted cuts due to the gRNA binding elsewhere than the intended target region $[27,85]$. This phenomenon is known to be accentuated in regions with sequences similar to the original sequence, e.g. paralogs genes or retrotransposons regions [24]. This feature, so far, severely hinders the technology to enter into advanced clinical phases. Screening every engineered cell for off-target effects after each genetic manipulation poses long term viability issues for the technology. Consequently, other technologies with proven track record such as TALENs are so far offering a safer solution for gene editing therapies. However, while CRISPR struggles as a standalone therapy, several efforts to minimize off-target cleavage have been reported. Recently, the development of an improved Cas9 variant with enhanced proofreading capacities has extensively reduced off-targeting effects while maintaining the high-cutting efficiency [9]. Additionally, powerful molecules with the ability to inactivate Cas proteins activity, named anti-CRISPR proteins, have been reported to significantly reduce off-targeting edits [89].

In the field of crop engineering, CRISPR techniques are currently having a major impact, facilitating cheaper, faster, applications. In green, companies applying CRISPR in the crop industry and biotech industry. In black, companies developing tools, cell lines and animal models

and more precise engineering in comparison to laborious and time-consuming traditional methods [3, 82]. However, it has yet to be determined whether CRISPR based gene editing of crops will be regulated the same way traditional genetically engineered crops are, which ultimately will settle its commercial value within this sector.

Currently, the industrial biotechnology field using metabolically engineered microbial cell factories is progressively shifting from studies with few genetic modifications to highly engineered strains. CRISPR has become a nearcommodity in the field as a result of the available panoply of engineering tools for these microbial cell factories, as well as the complex tasks these tools can perform. While most of the CRISPR proof-of-concepts have been carried out in well characterized industrial strains, more and more complex organisms successfully generating CRISPR/Cas9 mediated genome edits are being reported. A particular example concerns secondary metabolites, which are often derived from non-model organisms, thus making the corresponding biosynthetic pathways poorly characterized especially with the host being difficult to genetically engineer with traditional tools. In this example, one could either consider using 




Fig. 5 Map of key CRISPR players

CRISPR technology to integrate this large pathway into a well characterized organism, or, directly genetically engineer the host organism to further enhance the product formation or elucidate its idiosyncrasies $[52,75]$.

Acknowledgements The authors would like to thank Jens Christian Nielsen, Paulo Gonçalves Teixeira, and Philip Hemme for valuable feedbacks and discussions.

Funding This work was funded by the Novo Nordisk Foundation, Swedish Foundation for Strategic Research, Åforsk and the Knut and Alice Wallenberg foundation.

Open Access This article is distributed under the terms of the Creative Commons Attribution-NonCommercial 4.0 International License (http://creativecommons.org/licenses/by-nc/4.0/), which permits any noncommercial use, distribution, and reproduction in any medium, provided you give appropriate credit to the original author(s) and the source, provide a link to the Creative Commons license, and indicate if changes were made.

\section{References}

1. Bao Z, Xiao H, Liang J, Zhang L, Xiong X, Sun N, Si T, Zhao H (2014) Homology-integrated CRISPR-Cas (HI-CRISPR) system for one-step multigene disruption in Saccharomyces cerevisiae. ACS Synth Biol 4(5):585-594

2. Barrangou R, Fremaux C, Deveau H, Richards M, Boyaval P, Moineau S, Romero DA, Horvath P (2007) CRISPR provides acquired resistance against viruses in prokaryotes. Science 315(5819):1709-1712
3. Belhaj K, Chaparro-Garcia A, Kamoun S, Patron NJ, Nekrasov V (2015) Editing plant genomes with CRISPR/Cas9. Curr Opin Biotechnol 30(32):76-84

4. Bikard D, Marraffini LA (2013) Control of gene expression by CRISPR-Cas systems. F1000prime Rep 5

5. Bolotin A, Quinquis B, Sorokin A, Ehrlich SD (2005) Clustered regularly interspaced short palindrome repeats (CRISPRs) have spacers of extrachromosomal origin. Microbiology 151(8):2551-2561

6. Carroll D (2011) Genome engineering with zinc-finger nucleases. Genetics 188(4):773-782

7. Chavez A, Scheiman J, Vora S, Pruitt BW, Tuttle M, Iyer EP, Lin S, Kiani S, Guzman CD, Wiegand DJ, Ter-Ovanesyan D (2015) Highly efficient Cas9-mediated transcriptional programming. Nat Methods 12(4):326-328

8. Chavez A, Tuttle M, Pruitt BW, Ewen-Campen B, Chari R, Ter-Ovanesyan D, Haque SJ, Cecchi RJ, Kowal EJ, Buchthal J, Housden BE (2016) Comparison of Cas9 activators in multiple species. Nat Methods 13(7):563-567

9. Chen JS, Dagdas YS, Kleinstiver BP, Welch MM, Harrington LB, Sternberg SH, Joung JK, Yildiz A, Doudna JA (2017) Enhanced proofreading governs CRISPR-Cas9 targeting accuracy. Nature 550(7676):407

10. Cho JS, Choi KR, Prabowo CP, Shin JH, Yang D, Jang J, Lee SY (2017) CRISPR/Cas9-coupled recombineering for metabolic engineering of Corynebacterium glutamicum. Metab Eng 1(42):157-167

11. Cleto S, Jensen JV, Wendisch VF, Lu TK (2016) Corynebacterium glutamicum metabolic engineering with CRISPR interference (CRISPRi). ACS Synth Biol 5(5):375-385

12. Cong L, Ran FA, Cox D, Lin S, Barretto R, Habib N, Hsu PD, Wu X, Jiang W, Marraffini LA, Zhang F (2013) Multiplex genome engineering using CRISPR/Cas systems. Science 339(6121):819-823

13. Cress BF, Leitz QD, Kim DC, Amore TD, Suzuki JY, Linhardt RJ, Koffas MA (2017) CRISPRi-mediated metabolic engineering 
of $E$. coli for $O$-methylated anthocyanin production. Microb Cell Fact 16(1):10

14. Cress BF, Toparlak OD, Guleria S, Lebovich M, Stieglitz JT, Englaender JA, Jones JA, Linhardt RJ, Koffas MA (2015) CRISPathBrick: modular combinatorial assembly of type II-A CRISPR arrays for dCas9-mediated multiplex transcriptional repression in E. coli. ACS Synth Biol 4(9):987-1000

15. David F, Siewers V (2015) Advances in yeast genome engineering. FEMS Yeast Res 15(1):1-4

16. Deaner M, Alper HS (2017) Systematic testing of enzyme perturbation sensitivities via graded dCas9 modulation in Saccharomyces cerevisiae. Metab Eng 31(40):14-22

17. Deaner M, Mejia J, Alper HS (2017) Enabling graded and largescale multiplex of desired genes using a dual-mode dCas9 activator in Saccharomyces cerevisiae. ACS Synth Biol 6(10):1931-1943

18. Doench JG, Fusi N, Sullender M, Hegde M, Vaimberg EW, Donovan KF, Smith I, Tothova Z, Wilen C, Orchard R, Virgin HW (2016) Optimized sgRNA design to maximize activity and minimize off-target effects of CRISPR-Cas9. Nat Biotechnol 34(2):184-191

19. Doench JG, Hartenian E, Graham DB, Tothova Z, Hegde M, Smith I, Sullender M, Ebert BL, Xavier RJ, Root DE (2014) Rational design of highly active sgRNAs for CRISPR-Cas9mediated gene inactivation. Nat Biotechnol 32(12):1262-1267

20. Doudna JA, Charpentier E (2014) The new frontier of genome engineering with CRISPR-Cas9. Science 346.6213:1258096

21. Egelie KJ, Graff GD, Strand SP, Johansen B (2016) The emerging patent landscape of CRISPR-Cas gene editing technology. Nat Biotechnol 34(10):1025-1031

22. Farzadfard F, Perli SD, Lu TK (2013) Tunable and multifunctional eukaryotic transcription factors based on CRISPR/Cas. ACS Synth Biol 2(10):604

23. Fellmann C, Gowen BG, Lin PC, Doudna JA, Corn JE (2017) Cornerstones of CRISPR-Cas in drug discovery and therapy. Nat Rev Drug Discov 16(2):89-100

24. Ferreira R, Gatto F, Nielsen J (2017) Exploiting off-targeting in guide-RNAs for CRISPR systems for simultaneous editing of multiple genes. FEBS Lett

25. Ferreira R, Skrekas C, Nielsen J, David F (2017) Multiplexed CRISPR/Cas9 genome editing and gene regulation using Csy 4 in Saccharomyces cerevisiae. ACS Synth Biol

26. Fonfara I, Richter H, Bratovič M, Le Rhun A, Charpentier E (2016) The CRISPR-associated DNA-cleaving enzyme Cpf 1 also processes precursor CRISPR RNA. Nature 532(7600):517-521

27. Fu Y, Foden JA, Khayter C, Maeder ML, Reyon D, Joung JK, Sander JD (2013) High-frequency off-target mutagenesis induced by CRISPR-Cas nucleases in human cells. Nat Biotechnol 31(9):822-826

28. Fu Y, Sander JD, Reyon D, Cascio VM, Joung JK (2014) Improving CRISPR-Cas nuclease specificity using truncated guide RNAs. Nat Biotechnol 32(3):279-284

29. Gaj T, Gersbach CA, Barbas CF (2013) ZFN, TALEN, and CRISPR/Cas-based methods for genome engineering. Trends Biotechnol 31(7):397-405

30. Gander MW, Vrana JD, Voje WE, Carothers JM, Klavins E (2017) Digital logic circuits in yeast with CRISPR-dCas9 NOR gates. Nat Commun 8

31. Garst AD, Bassalo MC, Pines G, Lynch SA, Halweg-Edwards AL, Liu R, Liang L, Wang Z, Zeitoun R, Alexander WG, Gill RT (2017) Genome-wide mapping of mutations at single-nucleotide resolution for protein, metabolic and genome engineering. Nat Biotechnol 35(1):48-55

32. Gasiunas G, Barrangou R, Horvath P, Siksnys V (2012) Cas9crRNA ribonucleoprotein complex mediates specific DNA cleavage for adaptive immunity in bacteria. Proc Natl Acad Sci 109(39):E2579-E2586

33. Gilbert LA, Horlbeck MA, Adamson B, Villalta JE, Chen Y, Whitehead EH, Guimaraes C, Panning B, Ploegh HL, Bassik MC, Qi LS (2014) Genome-scale CRISPR-mediated control of gene repression and activation. Cell 159(3):647-661

34. Gilbert LA, Larson MH, Morsut L, Liu Z, Brar GA, Torres SE, Stern-Ginossar N, Brandman O, Whitehead EH, Doudna JA, Lim WA (2013) CRISPR-mediated modular RNA-guided regulation of transcription in eukaryotes. Cell 154(2):442-451

35. Gordon GC, Korosh TC, Cameron JC, Markley AL, Begemann MB, Pfleger BF (2016) CRISPR interference as a titratable, trans-acting regulatory tool for metabolic engineering in the cyanobacterium Synechococcus sp. strain PCC 7002. Metab Eng 30(38):170-179

36. Hilton IB, D'ippolito AM, Vockley CM, Thakore PI, Crawford GE, Reddy TE, Gersbach CA (2015 ) Epigenome editing by a CRISPR-Cas9-based acetyltransferase activates genes from promoters and enhancers. Nat Biotechnol 33(5):510-517

37. Hirano H, Gootenberg JS, Horii T, Abudayyeh OO, Kimura M, Hsu PD, Nakane T, Ishitani R, Hatada I, Zhang F, Nishimasu H (2016) Structure and engineering of Francisella novicida Cas9. Cell 164(5):950-961

38. Horwitz AA, Walter JM, Schubert MG, Kung SH, Hawkins K, Platt DM, Hernday AD, Mahatdejkul-Meadows T, Szeto W, Chandran SS, Newman JD (2015) Efficient multiplexed integration of synergistic alleles and metabolic pathways in yeasts via CRISPR-Cas. Cell Syst 1(1):88-96

39. Hsu PD, Lander ES, Zhang F (2014) Development and applications of CRISPR-Cas9 for genome engineering. Cell 157(6):1262-1278

40. Huang CH, Shen CR, Li H, Sung LY, Wu MY, Hu YC (2016) CRISPR interference (CRISPRi) for gene regulation and succinate production in cyanobacterium S. elongatus PCC 7942. Microb Cell Fact 15(1):196

41. Jakočiūnas T, Bonde I, Herrgård M, Harrison SJ, Kristensen M, Pedersen LE, Jensen MK, Keasling JD (2015) Multiplex metabolic pathway engineering using CRISPR/Cas9 in Saccharomyces cerevisiae. Metab Eng 31(28):213-222

42. Jakočiūnas T, Jensen MK, Keasling JD (2016) CRISPR/Cas9 advances engineering of microbial cell factories. Metab Eng 31(34):44-59

43. Jakočiūnas T, Jensen MK, Keasling JD (2017) System-level perturbations of cell metabolism using CRISPR/Cas9. Curr Opin Biotechnol 31(46): 134-140

44. Jensen ED, Ferreira R, Jakočiūnas T, Arsovska D, Zhang J, Ding L, Smith JD, David F, Nielsen J, Jensen MK, Keasling JD (2017) Transcriptional reprogramming in yeast using dCas9 and combinatorial gRNA strategies. Microb Cell Fact 16(1):46

45. Jessop-Fabre MM, Jakočiūnas T, Stovicek V, Dai Z, Jensen MK, Keasling JD, Borodina I (2016) EasyClone-MarkerFree: a vector toolkit for marker-less integration of genes into Saccharomyces cerevisiae via CRISPR-Cas9. Biotechnol J 11(8):1110-1117

46. Jinek M, Chylinski K, Fonfara I, Hauer M, Doudna JA, Charpentier E (2012) A programmable dual-RNA-guided DNA endonuclease in adaptive bacterial immunity. Science 337(6096):816-821

47. Joung JK, Sander JD (2013) TALENs: a widely applicable technology for targeted genome editing. Nat Rev Mol Cell Biol 14(1):49-55

48. Kaczmarzyk D, Cengic I, Yao L, Hudson EP (2017) Diversion of the long-chain acyl-ACP pool in Synechocystis to fatty alcohols through CRISPRi repression of the essential phosphate acyltransferase PlsX. Metab Eng 45:59-66 
49. Ke A, Ding F, Batchelor JD, Doudna JA (2007) Structural roles of monovalent cations in the HDV ribozyme. Structure 15(3):281-287

50. Kearns NA, Pham H, Tabak B, Genga RM, Silverstein NJ, Garber M, Maehr R (2015) Functional annotation of native enhancers with a Cas9-histone demethylase fusion. Nat Methods 12(5):401-403

51. Keren L, Zackay O, Lotan-Pompan M, Barenholz U, Dekel E, Sasson V, Aidelberg G, Bren A, Zeevi D, Weinberger A, Alon U (2013) Promoters maintain their relative activity levels under different growth conditions. Mol Syst Biol 9(1):701

52. Kim HU, Charusanti P, Lee SY, Weber T (2016) Metabolic engineering with systems biology tools to optimize production of prokaryotic secondary metabolites. Nat Product Rep 33(8):933-941

53. Kim SK, Han GH, Seong W, Kim H, Kim SW, Lee DH, Lee SG (2016) CRISPR interference-guided balancing of a biosynthetic mevalonate pathway increases terpenoid production. Metab Eng 30(38):228-240

54. Klann TS, Black JB, Chellappan M, Safi A, Song L, Hilton IB, Crawford GE, Reddy TE, Gersbach CA (2017) CRISPR-Cas9 epigenome editing enables high-throughput screening for functional regulatory elements in the human genome. Nat Biotechnol 35(6):561-568

55. Kleinstiver BP, Pattanayak V, Prew MS, Tsai SQ, Nguyen NT, Zheng Z, Joung JK (2016) High-fidelity CRISPR-Cas9 nucleases with no detectable genome-wide off-target effects. Nature 529(7587):490-495

56. Kleinstiver BP, Prew MS, Tsai SQ, Topkar VV, Nguyen NT, Zheng Z, Gonzales AP, Li Z, Peterson RT, Yeh JR, Aryee MJ (2015) Engineered CRISPR-Cas9 nucleases with altered PAM specificities. Nature 523(7561):481-485

57. Korkmaz G, Lopes R, Ugalde AP, Nevedomskaya E, Han R, Myacheva K, Zwart W, Elkon R, Agami R (2016) Functional genetic screens for enhancer elements in the human genome using CRISPR-Cas9. Nat Biotechnol 34(2):192-198

58. Kuivanen J, Wang YM, Richard P (2016) Engineering Aspergillus niger for galactaric acid production: elimination of galactaric acid catabolism by using RNA sequencing and CRISPR/Cas9. Microb Cell Fact 15(1):210

59. Larson MH, Gilbert LA, Wang X, Lim WA, Weissman JS, Qi LS (2013) CRISPR interference (CRISPRi) for sequence-specific control of gene expression. Nat Protoc 8(11):2180-2196

60. Li H, Shen CR, Huang CH, Sung LY, Wu MY, Hu YC (2016) CRISPR-Cas9 for the genome engineering of cyanobacteria and succinate production. Metab Eng 30(38):293-302

61. Li R, Li R, Li X, Fu D, Zhu B, Tian H, Luo Y, Zhu H (2017) Multiplexed CRISPR/Cas9-mediated metabolic engineering of $\gamma$-aminobutyric acid levels in Solanum lycopersicum. Plant Biotechnol J. https://doi.org/10.1111/pbi.12781

62. Li J, Meng X, Zong Y, Chen K, Zhang H, Liu J, Li J, Gao C (2016) Gene replacements and insertions in rice by intron targeting using CRISPR-Cas9. Nat Plants 12(2):16139

63. Li Y, Lin Z, Huang C, Zhang Y, Wang Z, Tang YJ, Chen T, Zhao X (2015) Metabolic engineering of Escherichia coli using CRISPR-Cas9 meditated genome editing. Metab Eng 30(31):13-21

64. Liang L, Liu R, Garst AD, Lee T, Beckham GT, Gill RT (2017) CRISPR EnAbled trackable genome engineering for isopropanol production in Escherichia coli. Metab Eng 31(41): 1

65. Liang Z, Chen K, Li T, Zhang Y, Wang Y, Zhao Q, Liu J, Zhang H, Liu C, Ran Y, Gao C (2017) Eicient DNA-free genome editing of bread wheat using CRISPR/Cas9 ribonucleoprotein complexes. Nat Commun 8:14261

66. Liu JJ, Kong II, Zhang GC, Jayakody LN, Kim H, Xia PF, Kwak S, Sung BH, Sohn JH, Walukiewicz HE, Rao CV (2016)
Metabolic engineering of probiotic Saccharomyces boulardii. Appl Environ Microbiol 82(8):2280-2287

67. Liu Q, Gao R, Li J, Lin L, Zhao J, Sun W, Tian C (2017) Development of a genome-editing CRISPR/Cas9 system in thermophilic fungal Myceliophthora species and its application to hypercellulase production strain engineering. Biotechnol Biofuels 10(1): 1

68. Lv L, Ren YL, Chen JC, Wu Q, Chen GQ (2015) Application of CRISPRi for prokaryotic metabolic engineering involving multiple genes, a case study: controllable P (3HB-co-4HB) biosynthesis. Metab Eng 31(29):160-168

69. Makarova KS, Wolf YI, Alkhnbashi OS, Costa F, Shah SA, Saunders SJ, Barrangou R, Brouns SJ, Charpentier E, Haft DH, Horvath P (2015) An updated evolutionary classification of CRISPRCas systems. Nat Rev Microbiol 13(11):722-736

70. Mali P, Yang L, Esvelt KM, Aach J, Guell M, DiCarlo JE, Norville JE, Church GM (2013) RNA-guided human genome engineering via Cas9. Science 339(6121):823-826

71. Marraffini LA, Sontheimer EJ (2010) CRISPR interference: RNA-directed adaptive immunity in bacteria and archaea. Nat Rev Genet 11(3):181-190

72. Meng J, Feng R, Zheng G, Ge M, Mast Y, Wohlleben W, Gao J, Jiang W, Lu Y (2017) Improvement of pristinamycin I (PI) production in Streptomyces pristinaespiralis by metabolic engineering approaches. Synth Syst Biotechn

73. Mullard A (2017) FDA approves first CAR T therapy. Nat Rev Drug Discov 16(10):669

74. Mullard A (2017) Second anticancer CAR T therapy receives FDA approval. Nat Rev Drug Discov 16(12):818

75. Nielsen JC, Nielsen J (2017) Development of fungal cell factories for the production of secondary metabolites: linking genomics and metabolism. Synth Syst Biotechnol 2:5-12. https://doi. org/10.1016/j.synbio.2017.02.002

76. Nissim L, Perli SD, Fridkin A, Perez-Pinera P, Lu TK (2014) Multiplexed and programmable regulation of gene networks with an integrated RNA and CRISPR/Cas toolkit in human cells. Mol Cell 54(4):698-710

77. Ozaki A, Konishi R, Otomo C, Kishida M, Takayama S, Matsumoto T, Tanaka T, Kondo A (2017) Metabolic engineering of Schizosaccharomyces pombe via CRISPR-Cas9 genome editing for lactic acid production from glucose and cellobiose. Metab Eng Commun 1(5):60-67

78. Perez-Pinera P, Kocak DD, Vockley CM, Adler AF, Kabadi AM, Polstein LR, Thakore PI, Glass KA, Ousterout DG, Leong KW, Guilak F (2013) RNA-guided gene activation by CRISPR-Cas9based transcription factors. Nat Methods 10(10):973-976

79. Qi LS, Larson MH, Gilbert LA, Doudna JA, Weissman JS, Arkin AP, Lim WA (2013) Repurposing CRISPR as an RNA-guided platform for sequence-specific control of gene expression. Cell 152(5):1173-1183

80. Ran FA, Hsu PD, Lin CY, Gootenberg JS, Konermann S, Trevino AE, Scott DA, Inoue A, Matoba S, Zhang Y, Zhang F (2013) Double nicking by RNA-guided CRISPR Cas9 for enhanced genome editing specificity. Cell 154(6):1380-1389

81. Ren J, Zhao Y (2017) Advancing chimeric antigen receptor T cell therapy with CRISPR/Cas9. Protein Cell. 22:1

82. Rodríguez-Leal D, Lemmon ZH, Man J, Bartlett ME, Lippman ZB (2017) Engineering quantitative trait variation for crop improvement by genome editing. Cell 171(2):470-480

83. Ryan OW, Skerker JM, Maurer MJ, Li X, Tsai JC, Poddar S, Lee ME, DeLoache W, Dueber JE, Arkin AP, Cate JH (2014) Selection of chromosomal DNA libraries using a multiplex CRISPR system. Elife 19(3):e03703

84. Sapranauskas R, Gasiunas G, Fremaux C, Barrangou R, Horvath P, Siksnys V (2011) The Streptococcus thermophilus CRISPR/ 
Cas system provides immunity in Escherichia coli. Nucl Acids Res 39(21):9275-9282

85. Schaefer KA, Wu WH, Colgan DF, Tsang SH, Bassuk AG, Mahajan VB (2017) Unexpected mutations after CRISPR-Cas9 editing in vivo. Nat Methods 14(6):547-548

86. Shen JP, Zhao D, Sasik R, Luebeck J, Birmingham A, BojorquezGomez A, Licon K, Klepper K, Pekin D, Beckett AN, Sanchez KS (2017) Combinatorial CRISPR-Cas9 screens for de novo mapping of genetic interactions. Nat Methods

87. Sheridan C (2017) CRISPR patent estate splinters. Nat Biotechnol 35(9):808-809

88. Shi S, Liang Y, Zhang MM, Ang EL, Zhao H (2016) A highly efficient single-step, markerless strategy for multi-copy chromosomal integration of large biochemical pathways in Saccharomyces cerevisiae. Metab Eng 31(33):19-27

89. Shing J, Jiang F, Liu JJ, Bray NL, Rauch BJ, Baik SH, Nogales E, Bondy-Denomy J, Corn JE, Doudna JA (2017) Disabling Cas9 by an anti-CRISPR DNA mimic. bioRxiv 129627

90. Siksnys V, Gasiunas G (2016) Rewiring Cas9 to target new PAM sequences. Mol Cell 61(6):793-794

91. Smith JD, Suresh S, Schlecht U, Wu M, Wagih O, Peltz G, Davis RW, Steinmetz LM, Parts L, Onge RP (2016) Quantitative CRISPR interference screens in yeast identify chemical-genetic interactions and new rules for guide RNA design. Genome Biol 17(1):45

92. Stella S, Alcon P, Montoya G (2017) Class 2 CRISPR-Cas RNAguided endonucleases: Swiss Army knives of genome editing. Nat Struct Mol Biol 24(11):882

93. Stovicek V, Borodina I, Forster J (2015) CRISPR-Cas system enables fast and simple genome editing of industrial Saccharomyces cerevisiae strains. Metab Eng Commun 31(2):13-22

94. Vanegas KG, Lehka BJ, Mortensen UH (2017) SWITCH: a dynamic CRISPR tool for genome engineering and metabolic pathway control for cell factory construction in Saccharomyces cerevisiae. Microb Cell Fact 16(1):25
95. Wang H, La Russa M, Qi LS (2016) CRISPR/Cas9 in genome editing and beyond. Annu Rev Biochem 2(85):227-264

96. Wen Z, Minton NP, Zhang Y, Li Q, Liu J, Jiang Y, Yang S (2017) Enhanced solvent production by metabolic engineering of a twinclostridial consortium. Metab Eng 31(39):38-48

97. Westbrook AW, Moo-Young M, Chou CP (2016) Development of a CRISPR-Cas9 tool kit for comprehensive engineering of Bacillus subtilis. Appl Environ Microbiol 82(16):4876-4895

98. Wolter F, Puchta H (2017) Knocking out consumer concerns and regulator's rules: efficient use of CRISPR/Cas ribonucleoprotein complexes for genome editing in cereals. Genome Biol 18(1):43

99. Wu MY, Sung LY, Li H, Huang CH, Hu YC (2017) Combining CRISPR and CRISPRi systems for metabolic engineering of E. coli and 1, 4-BDO biosynthesis. ACS Synth Biol 6(12):2350-2361

100. Zalatan JG, Lee ME, Almeida R, Gilbert LA, Whitehead EH, La Russa M, Tsai JC, Weissman JS, Dueber JE, Qi LS, Lim WA (2015) Engineering complex synthetic transcriptional programs with CRISPR RNA scaffolds. Cell 160(1):339-350

101. Zetsche B, Gootenberg JS, Abudayyeh OO, Slaymaker IM, Makarova KS, Essletzbichler P, Volz SE, Joung J, van der Oost J, Regev A, Koonin EV (2015) Cpf1 is a single RNA-guided endonuclease of a class 2 CRISPR-Cas system. Cell 163(3):759-771

102. Zhang B, Liu ZQ, Liu C, Zheng YG (2016) Application of CRISPRi in Corynebacterium glutamicum for shikimic acid production. Biotech Lett 38(12):2153-2161

103. Zhu X, Zhao D, Qiu H, Fan F, Man S, Bi C, Zhang X (2017) The CRISPR/Cas9-facilitated multiplex pathway optimization (CFPO) technique and its application to improve the Escherichia coli xylose utilization pathway. Metab Eng 1(43):37-45 